\title{
Vibrationen und Kieferorthopädie
}

DiBiase, Andrew ; Papageorgiou, Spyridon N ; Cobourne, Martyn

DOI: https://doi.org/10.1055/s-0043-113334

Other titles: Vibrations and Orthodontics

Posted at the Zurich Open Repository and Archive, University of Zurich ZORA URL: https://doi.org/10.5167/uzh-149463

Journal Article

Accepted Version

Originally published at:

DiBiase, Andrew; Papageorgiou, Spyridon N; Cobourne, Martyn (2017). Vibrationen und Kieferorthopädie. Informationen aus Orthodontie und Kieferorthopädie, 49(02):153-156.

DOI: https://doi.org/10.1055/s-0043-113334 


\section{Vibrational force and orthodontics}

Andrew T. DiBiase ${ }^{1}$, Spyridon N. Papageorgiou ${ }^{2}$ and Martyn T. Cobourne ${ }^{3}$

${ }^{1}$ Department of Orthodontics, William Harvey Hospital, East Kent Hospitals University NHS Foundation Trust, Ashford, United Kingdom

${ }^{2}$ Clinic of Orthodontics and Pediatric Dentistry, Center of Dental Medicine, University of Zurich, Zurich, Switzerland

${ }^{3}$ Department of Orthodontics, King's College London Dental Institute, Hon consultant in orthodontics, Guy's and St Thomas' NHS Foundation Trust, London, United Kingdom

\section{Manuscript}

Therapies involving vibration as a sole or adjunct medium have a long history in health care. It was 1895 when Dr. John Harvey Kellogg suggested that vibration could be used to improve the blood circulation and relieve constipation. Subsequently, the use of whole body vibrational therapy has been implemented to a wide variety of conditions, including cerebral palsy ${ }^{1,2}$, Parkinson's disease ${ }^{3}$, diabetes ${ }^{4}$, obesity ${ }^{5}$, stroke ${ }^{6}$, back pain ${ }^{7}$, and osteoporosis ${ }^{8}$. In relation to the last, it has been widely recognized for over a century that environmental conditions can directly influence bone mineral density, in terms of a load-induced bone remodeling ${ }^{9}$. This forms the basic principle of orthodontic tooth movement and is the reason why significant bone loss is observed in astronauts exposed to extended periods of microgravity ${ }^{10}$. Therapeutical high-frequency low-magnitude mechanical stimulation can be effective in increasing bone and muscle mass following prolonged loss of functional weightbearing ${ }^{11,12}$. Vibrational therapy has also been shown to increase bone density in other patient groups prone to bone loss, such as post-menopausal women and disabled ambulant children ${ }^{13,14}$.

In animal models cyclic bone loading appears to promote craniofacial suture growth and remodeling ${ }^{15,16}$. For example, vibrational stimulation in rats has been associated with increased rate of orthodontic expansion and increased rate of space closure, as well as increased osteoclastic activity in the periodontal ligament ${ }^{17,18}$.

Based on this biologic principle, several appliances have been developed and are now commercially available that are designed to deliver a vibrational force directly to the dentition in patients undergoing orthodontic treatment. The Tooth Masseuse delivers a force of $0.06 \mathrm{~N}$ at a frequency of $111 \mathrm{~Hz}$, the daily use of which should - according to the manufacturer-- 
reduce pain and discomfort during orthodontic treatment. The AcceleDent appliance is a hands-free device consisting of an activator unit into which is inserted a removable occlusal wafer, onto which the patient bites. The unit vibrates, delivering a force of $0.2 \mathrm{~N}$ at a frequency of $30 \mathrm{~Hz}$ to the dentition with a daily use of 20 minutes per day. Claims by the manufacturer include not only a reduction in the levels of pain and discomfort, but also an increased speed of tooth movement, thereby reducing overall orthodontic treatment time. The most recent appliance to appear on the market is the Propel ProV, which is again a hands free appliance, with an occlusal splint onto which the patient bites. The usage time has now been reduced to 5 minutes a day, which again purports to significantly speed up orthodontic tooth movement and therefore reduce treatment time.

The first clinical research investigating the use supplemental vibratory force in orthodontics was retrospective and promisingly showed a relative increase rate of tooth movement in a small case series of 14 patients, using an early version of the AcceleDent appliance ${ }^{19}$. Anotherretrospective study evaluated the effect of the AcceleDent on the rate of levelling and aligning in the lower arch with fixed appliances on a cohort of consecutively treated Class II cases, undergoing simultaneous molar distalisation with an intraoral device supported by mini-screws ${ }^{20}$. From the 67 included patients, 30 received supplementary daily use of the AcceleDent appliance, while 37 acted as controls with no supplementary vibration. The mean times for both alignment and levelling were significantly faster in the AcceleDent group that used the appliance. However, this study was retrospective in nature there is an increased riskthere is risk for exaggeration of the treatment effects due to bias. Also the baseline characteristics such as the initial crowding, age and gender were reported incompletely and not accounted for in the statistical analysis. It is therefore impossible to conclude as to whether the differences found were due to the use of vibrational force or to baseline confounding. Finally, no sample size calculation was carried a priori, which resulted in the study being underpowered.

A subsequent publication from this study reported an increased rate of molar distalisation in the group using the AcceleDent appliance ${ }^{21}$. Measurements were made to the maxillary first molars using lateral cephalograms, which is prone to high levels of indication errors due to superimposition of the right and left sides. Also the statistically significant differences reported in this study only related to the distalisation of molar root apices, but not the crowns, which makes them of questionable clinical relevance. Combined these factors make it difficult to draw any robust conclusions from this study. 
Subsequent prospective clinical research reported deviating results on the treatment effects of supplementary vibration. One Randomized Clinical Trial (RCT) showed a significant increase in miniscrew-supported maxillary canine retraction rate with the use of the AcceleDent appliance compared to a non-active shame device ${ }^{22}$. However, in this study, large dropout rates were observed, patients of very wide age range were included, while the measurements appear to have been taken directly intraorally with no reproducibility reported. The reported differences,while statistically significant, were small in magnitude and therefore it is debatable whether they are of clinically significance.

A further RCT also reported increased rate of maxillary canine retraction rate using vibratory force ${ }^{23}$. In this study the vibratory force was delivered using an electric toothbrush applied directly to the canine allocated to the study group in a split mouth design and therefore this does not correspond to existing commercial vibration appliances. The sample sizes in this study were very small, with no evidence of a priori sample size calculation, and as with the previous study, while the differences in canine retraction were statistically significant, their clinical relevance is doubtful.

The initial encouraging results of these studies have not been supported by subsequent RCTs investigating the rate of tooth movement during the initial alignment phase of fixed appliance therapy. One study carried out in Australia reported on the alignment speed in 66 patients randomized to either fixed appliances alone or fixed appliances supplemented with daily use of the Tooth Mausseuse ${ }^{24}$. The groups were investigated during the initial 10 week alignment period with $0.014 "$ nickel-titanium archwires and no difference was found between them. The same research group undertook a further study using the AcceleDent appliance on 40 Class II patients, looking at the alignment in the mandibular $\operatorname{arch}^{25}$. The patients were randomized either to fixed appliances alone or fixed appliances with daily use of the AcceleDent appliance. They were followed up for a 10 week period. Again, no difference was found between the two groups in speed of alignment or arch perimeter changes during this period. They also asked the patients to record pain experienced using during treatment with a visual analogue scale and consumption of analgesics. While there was no difference in perceived pain, the group using the AcceleDent appliance reported less use of analgesics on day 1 of the trial.

The possible benefits of vibrational force in terms of reduced pain and discomfort have been also reported in another $\mathrm{RCT}^{26}$. In this study 70 patients were were allocated into either the control group or the study groups that were instructed to use the AcceleDent appliance for 20 minutes each day. The were subsequently asked to keep a pain diary for the first seven 
days following each appointment when their appliances were adjusted and then weekly after that, noting both overall pain and pain on biting. The patients were seen monthly for the 4 month period over which the study was carried out, and were instructed not to take any painkillers. Again, pain was self-reported using a visual analogue scale and the four weekly pain scores were averaged out to give a monthly score. For the 58 patients reported on from the original 70, both for biting and overall pain in the AcceleDent group reported lower levels of pain at all time points. However, the baseline demographics of the groups at the start of the study including age, sex, type of malocclusion, or degree of crowding were not reported at all. Additionally, no information was provided regarding the used appliances or archwire sequence. All these factors may well have affected perceived pain levels and should have been accounted for, in order to link any differences between the two groups to the use of the AcceleDent appliance.

A larger RCT was carried out in the UK looking both at alignment speed and pain during fixed appliance phase with the use of the AcceleDent appliance ${ }^{27,28}$. This was a three-arm parallel RCT looking at adolescents with significant crowding in the mandibular arch whose treatment involved the extraction of lower premolars. They were treated with a 0.022 " slot pre-adjusted edgewise appliance and randomly allocated into one of three groups: fixed appliances alone, fixed appliances plus daily use of a functional AcceleDent appliance and fixed appliances plus daily use of a non-functional AcceleDent appliance. A total of 81 subjects were recruited and followed through the alignment phase of treatment with the same archwire sequence: $0.014^{\prime}$, $0.018^{\prime \prime}$ and $0.018^{\prime \prime} \times 0.025^{\prime \prime}$ nickel titanium, and 0.019" x 0.025" stainless steel. Speed of alignment was measured from serial study casts of the lower arch using Little's irregularity index. Interestingly, no difference was found in this study for either initial speed of alignment on placing the $0.014^{\prime \prime}$ nickel titanium wires or overall speed of alignment over the whole study period until $0.019^{\prime \prime} \times 0.025^{\prime \prime}$ stainless steel wires were placed. The only difference found was that the greater the initial irregularity was, the faster the initial and overall speed of alignment-something that is to be expected.

This study also looked at the level of pain experienced and the use of analgesics during the initial alignment. The patients were asked to record their experience of pain using a visual analogue score at set time points during the first week following placement of 0.014 " and 0.018 nickel titanium wire. They were also asked to keep a record of any analgesics they used. All three groups showed a similar pattern with pain peaking at 24 hours following placement of the wire and then subsiding to baseline levels over the next week. After accounting for all confounders, there was no significant difference in the mean maximum pain experienced 
between the three groups. The only difference found was for age, with younger patients reporting higher levels of pain. Similarly the use of the AcceleDent appliance had no impact on the use of painkillers, while only a directly proportional relationship between analgesic use and pain levels was found.

Finally, the same RCT reported on levels of external apical root resorption ${ }^{29}$, being measured from long cone periapical radiographs of the upper right central incisor taken at the start of treatment and following placement of a 0.019 " x 0.025 stainless steel. Again, no differences were found between the three groups, although this was a secondary outcome of the study and was probably underpowered.

The findings of this study therefore support the results of the studies carried out by the research team in Australia, but do not confirm the results of initial studies reporting benefits both in relation to speed of alignment and pain experience. However, these studies were either retrospective or had serious methodological issues, particularly in relation to sample size, baseline equivalence between groups, randomization methods, and incomplete reporting, which make them susceptible to bias and their results should be interpreted with caution.

On the contrary, in the studies carried out in the UK and Australia, particularly in relation the AcceleDent appliance, patients were appropriately allocated prospectively and in a random fashion, making them a priori comparable for age, sex, and the irregularity. There was minimal drop out and therefore adequate power was retained. The UK study also included a sham nonvibrating device to account for any placebo effects.

Like all real world studies they were without issues. In the UK study it was impossible to completely blind the groups as the non-functional AcceleDent device looked like the functional one, but did not vibrate. Also, the authors reported that the timers on the devices proved inaccurate and therefore they data pertaining appliance usage could not be robustly obtained. It is therefore possible that the negative findings are a result of patients not using the AcceleDent appliance as instructed. However, as patients were randomized across groups, a similar proportion of compliant and non-compliant patients is expected for each group. Additionally, the studies were carried out during the initial stages of treatment when compliance tends to be optimal and subjects were monitored carefully, being asked to bring the appliance to all their appointments when they were inspected for use. Managing issues of compliance is a part of everyday practice, making the results of this study applicable to "real world" orthodontics. Interestingly, compliance was recorded in the second study from the Australian team, who reported similar negative results for both pain and speed of alignment. 
Therefore the authors feel that these three studies offer the highest quality evidence available to date.

In conclusion, based on the current available evidence the use supplemental vibration force during orthodontic treatment with fixed appliances does not increase the rate of tooth movement or reduce the level of pain reported during the initial alignment phases of treatment. It is important that in a world when treatment techniques and modalities are heavily marketed -and often directly to patients-- clinicians and patients are aware of the best available evidence to allow them to make properly informed choices about their treatment. 


\section{References}

1. Park C, Park ES, Choi JY, Cho Y, Rha DW . Immediate Effect of a Single Session of Whole Body Vibration on Spasticity in Children With Cerebral Palsy. Ann Rehabil Med. 2017 Apr;41(2):273-278.

2. Ko MS, Sim YJ, Kim DH, Jeon HS, Effects of Three Weeks of Whole-Body Vibration Training on Joint-Position Sense, Balance, and Gait in Children with Cerebral Palsy: A Randomized Controlled Study Physiother Can. 2016; 68(2): 99-105.

3. King LK, Almeida QJ, Ahonen H. Short-term effects of vibration therapy on motor impairments in Parkinson's disease. NeuroRehabilitation. 2009;25(4):297-306.

4. Zhang J, Zhang H, Kan L, Zhang C, Wang P. The effect of whole body vibration therapy on the physical function of people with type II diabetes mellitus: a systematic review J Phys Ther Sci. 2016; 28(9): 2675-2680.

5. Alvarez-Alvarado S, Jaime SJ , Ormsbee MJ , Campbell JC, Post J , Pacilio J , Figueroa A. Benefits of whole-body vibration training on arterial function and muscle strength in young overweight/obese women. Hypertens Res. 2017;40(5):487-492.

6.Choi ET, Kim YN, Cho SW, Lee DK. The effects of visual control whole body vibration exercise on balance and gait function of stroke patients J Phys Ther Sci. 2016; 28(11): 3149-3152.

7. Wang XQ, Pi YL, Chen PJ, Chen BL, Liang LC, Li X, Wang X, Zhang J. Whole body vibration exercise for chronic low back pain: study protocol for a single-blind randomized controlled trial Trials 2014, 15:104

8. Fratini A, Bonci T, Bull AMJ (2016) Whole Body Vibration Treatments in Postmenopausal Women Can Improve Bone Mineral Density: Results of a Stimulus Focussed Meta-Analysis. PLoS ONE 11(12): e0166774. doi:10.1371/journal. pone.0166774

9. Wolff, J. (1892) The Law of Bone Remodeling (original publication 1892 translated in 1986 by P. Maquet and R. Furlong): Springer, Berlin.

10. A. LeBlanc1, V. Schneider2, L. Shackelford2, S. West1, V. Oganov3, A. Bakulin3, L. 
Voronin3Bone mineral and lean tissue loss after long duration space flight J Musculoskel Neuron Interact 2000; 1(2):157-160

11. Holguin N, Muir J, Rubin C, Judex S. Short applications of very low-magnitude vibrations attenuate expansion of the intervertebral disc during extended bed rest. Spine J 2009;9:470-477.

12. Wang $H$, Wan $Y$, Tam KF, Ling S, Bai Y, Deng Y, Liu Y, Zhang H, Cheung WH, Qin L, et al. Resistive vibration exercise retards bone loss in weight-bearing skeletons during 60 days bed rest. Osteoporosis Int 2012;23:2169-2178.

13. Rubin C, Recker R, Cullen D, Ryaby J, McCabe J, McLeod K. Prevention of postmenopausal bone loss by a low-magnitude, high-frequency mechanical stimuli: a clinical trial assessing compliance, efficacy, and safety. J Bone Miner Res2004;19:343-351.

14. Ward K, Alsop C, Caulton J, Rubin C, Adams J, Mughal Z. Low magnitude mechanical loading is osteogenic in children with disabling conditions. J Bone Miner Res 2004;19:360-369.

15. Mao JJ, Wang X, Mooney MP, Kopher RA, Nudera JA. Strain induced osteogenesis of the craniofacial suture upon controlled delivery of lowfrequency cyclic forces. Front Biosci 2003;8:a10-a7.

16. Peptan Al, Lopez A, Kopher RA, Mao JJ. Responses of intramembranous bone and sutures upon in vivo cyclic tensile and compressive loading. Bone 2008;42(2):432-438.

17. Darendeliler MA, Zea A, Shen G, Zoellner H. Effects of pulsed electromagnetic field vibration on tooth movement induced by magnetic and mechanical forces: a preliminary study. Aust Dent J 2007;52:282-287. 
18. Nishimura M, Chiba M, Ohashi T, Sato M, Shimizu Y, Igarashi K, Mitani H. Periodontal tissue activation by vibration: intermittent stimulation by resonance vibration accelerates experimental tooth movement in rats. Am J Orthod Dentofacial Orthop 2008;133:572-583.

19. Kau CH, Nguyen JT, English JD. The clinical evaluation of a novel cyclical force generating device in orthodontics. Orthod Prac US 2010;1(1):10-15.

20. Bowman SJ. The effect of vibration on the rate of leveling and alignment. J Clin Orthod 2014;48(11):678-688.

21. Bowman SJ. The Effect of Vibration on Molar Distalization. J Clin Orthod 2016;50:683-693

22. Pavlin D, Anthony R, Raj V, Gakunga PT. Cyclic loading (vibration) accelerates tooth movement in orthodontic patients: A double-blind randomized controlled trial. Semin Orthod 2015;21(3):187-194.

23. Leethanakul C, Suamphan S, Jitpukdeebodintra S, Thongudomporn U, Charoemratrote C. Vibratory stimulation increases interleukin-1 beta secretion during orthodontic tooth Angle Orthod 2016;86:74-80.

24. Miles $\mathrm{P}$, Smith $\mathrm{H}$, Weyant $\mathrm{R}$, Rinchuse DJ. The effects of a vibrational appliance on tooth movement and patient discomfort: A prospective randomized clinical trial. Aust Orthod J 2012;28(2):213-218.

25. Miles P, Fisher E. Assessment of the changes in arch perimeter and irregularity in the mandibular arch during initial alignment with the AcceleDent Aura appliance vs no appliance in adolescents: A single-blind randomized clinical trial. Am J Orthod Dentofacial Orthop 2016;150:928-36.

26. Lobre WD, Callegari BJ, Gardner G, Marsh CM, Bush AC, Dunn WJ. Pain control in orthodontics using a micropulse vibration device: A randomized clinical trial. Angle Orthod 2016;86(4);625-630.

27. Woodhouse NR, DiBiase AT, Johnson N, Slipper C, Grant J, Alsaleh M, Donaldson AN, 
Cobourne MT. Supplemental vibrational force during orthodontic alignment: A randomized trial. J Dent Res 2015a;94(5): 682-689.

28. Woodhouse NR, DiBiase AT, Papageorgiou SN, Johnson N, Slipper C, Grant J, Alsaleh M, Cobourne MT. Supplemental vibrational force does not reduce pain experience during initial alignment with fixed orthodontic appliances: A multicenter randomized clinical trial. Sci Rep 2015b;5:17224.

29. DiBiase AT, Woodhouse NR, Papageorgiou SN, Johnson N, Slipper C, Grant J, Alsaleh M, Cobourne MT. Effect of supplemental vibrational force on orthodontically induced inflammatory root resorption: A multicenter randomized clinical trial. Am J Orthod Dentofacial Orthop 2016;150:918-927. 